\title{
Retinopatía del prematuro: resultados de un programa de prevención, detección y tratamiento
}

\author{
Retinopathy of prematurity: results of a prevention, \\ detection and treatment program
}

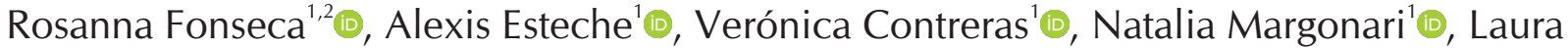

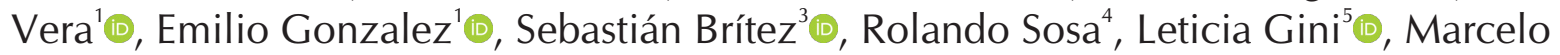

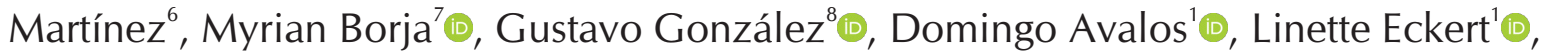 \\ Nicolas González ${ }^{1}$ (D)

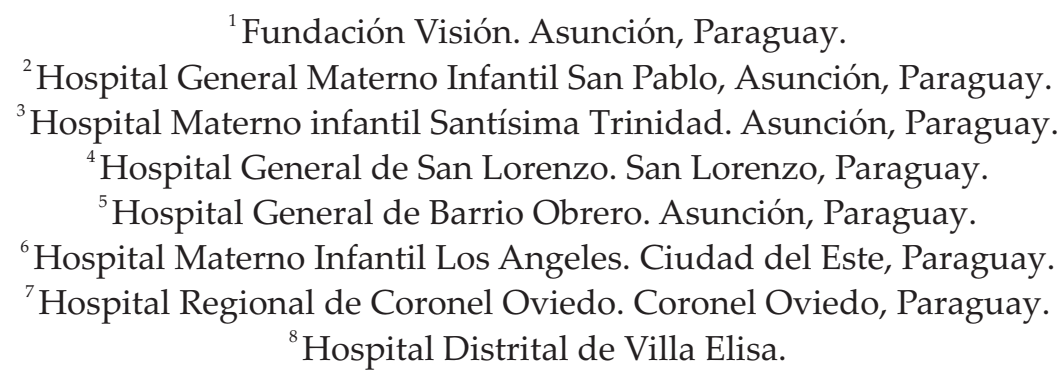

\section{RESUMEN}

Introducción: La Retinopatía del Prematuro (ROP) es una de las principales causas de ceguera prevenibles en la infancia. La Fundación Visión implementa; el Programa de Prevención, Detección y Tratamiento en el año 2015. Objetivos: Describir los resultados de implementación y los resultados alcanzados por un programa de ROP en su accesibilidad, efectividad y calidad de atención. Materiales y Métodos: Estudio descriptivo, ambispectivo, de casos consecutivos de recién nacidos prematuros con criterios de evaluación; de una población evaluada en el año 2009 y del 2015 al 2019. Resultados: Las unidades neonatales incluidas aumentaron de 3 a 7; cubriendo Asunción, área Central, Caaguazú y Alto Paraná. De los registros se constato que la cobertura aumento de 36\% en el año 2009 a 97\% en el 2019. Desde que se instaló el programa; en el primer año la proporción del número de evaluaciones aumento entre el 28 y el 218\%; y los resultados globales de julio 2015 a diciembre 2019 fueron: número totales de pacientes con criterio: 2397 pacientes; número totales de pacientes evaluados: 2080 (86,8\%), número de pacientes con ROP: 416/2080 pacientes (20\%), número de pacientes con ROP que requirieron tratamiento: $76 / 416$ pacientes $(18,2 \%)$, proporción global de

\section{ABSTRACT}

Introduction: Retinopathy of Prematurity (ROP) is one of the main preventable causes of blindness in childhood. The Vision Foundation implemented the Prevention, Detection and Treatment Program in 2015. Objective: To describe the implementation results and the outcomes achieved by a ROP program regarding its accessibility, effectiveness and quality of care. Materials and Methods: This was a descriptive and ambispective study of consecutive cases of premature newborns with criteria for evaluation, of a population evaluated in the year 2009 and from 2015 to 2019. Results: The participating neonatal units increased from 3 to 7; covering Asunción, the Central, Caaguazú and Alto Paraná Departments. From the records, it was found that program coverage increased from 36\% in 2009 to $97 \%$ in 2019. Since the beginning of the program, during the first year the proportion of the number of evaluations increased between 28 and 218\%; the global results from July 2015 to December 2019 were: total number of patients with criteria: 2397 patients; total number of patients evaluated: 2,080 $(88 \%)$, number of patients with ROP: $416 / 2080$ patients (20 $\%)$, number of patients with ROP requiring treatment: $76 / 416$ patients $(18.3 \%)$, overall proportion of severe ROP

Correspondencia: Rosanna Fonseca Correo: rosannafonseca74@gmail.com Conflictos de interés: Los autores declaran no presentar conflicto de interés. 
ROP grave con tratamiento: $76 / 2080$ pacientes $(3,4 \%)$. Conclusiones: El programa a través multidisciplinario innovación proporción con telemedicina logró aumentar la proporcion de cobertura y la disminución de los casos graves que requirieron tratamiento.

Palabras claves: Retinopatía del Prematuro, prematurez, epidemiología.

\section{INTRODUCCIÓN}

Por diversas razones; ya sean estas; políticas, socioculturales y/o económicas, en Latinoamérica los tipos de indicadores de salud evaluados; los tiempos en la innovación de tratamientos, de tecnologías y la formación de especialistas van más retrasados en relación a países más desarrollados; a medida que la mortalidad neonatal pasó a constituir el principal componente de la mortalidad infantil se replantearon los indicadores de salud y por ende el tipo y el enfoque de las intervenciones en los países en vías de desarrollo ${ }^{(1-3)}$.

En Paraguay ante esa nueva situación de salud pública se sumaron esfuerzos establecidos a través del Plan Estratégico Nacional; buscando mejorar los indicadores neonatales tanto de mortalidad como de morbilidades ${ }^{(4)}$.

La retinopatía del prematuro (ROP) constituye una de las principales morbilidades observadas; siendo un indicador de calidad de atención neonatal; y es definido muchas veces como una ventana del neurodesarrollo neonatal; y constituye una de las principales causas de ceguera infantil prevenible a nivel mundial ${ }^{(5,6)}$.

Es así que se considera que en Latinoamérica nos encontramos en la tercera epidemia de ROP; desde principios del año 2000; porque si bien fueron aumentando las unidades neonatales y por ende la sobrevida de niños prematuros; estos lugares se caracterizan por una variación en la calidad de la atención neonatal y una cobertura inadecuada de detección y tratamiento de la retinopatía del prematuro, observándose ROP tanto en los recién nacidos ( $R N)$ extremadamente prematuros como en with treatment: $76 / 2080$ patients (3.4\%). Conclusions: The program, using a multidisciplinary team and the innovation of telemedicine, managed to increase the proportion of coverage and the reduction of serious cases that require treatment.

Key words: Retinopathy of Prematurity, prematurity, epidemiology.

aquellos prematuros más grandes y maduros ${ }^{(6)}$.

Por lo que la retinopatía del prematuro no sólo se encuentra entre las causas más frecuentes de ceguera adquirida en los niños, sino que también es un excelente indicador de atención en un establecimiento de salud ${ }^{(7,8)}$.

Constituye ante lo expuesto de gran importancia que cada región y país establezcan sus protocolos de prevención, detección y tratamiento de ROP; con el fin de mejorar los indicadores de atención neonatal; conocer la incidencia de la patología a manera de establecer estrategias de evaluación e intervenir oportunamente según los hallazgos. Los criterios de evaluación varían según las regiones; por recomendaciones de la Organización Mundial de la Salud para Latinoamérica se recomienda evaluar RN prematuros menores de 32 semanas de edad gestacional, y/o con menos de 1500 gramos de peso al nacer, y/o RN prematuros con edades gestacionales comprendidas entre las 33 y las 36 semanas inclusive, de cualquier peso al nacer, que hayan requerido oxígeno o presenten otros factores de riesgo para presentar retinopatía de la prematuridad en algún momento entre su nacimiento y el egreso hospitalario $^{(7,9,10)}$.

Se espera que a medida que mejore la calidad de la atención neonatal, los riesgos a que un RN prematuro relativamente maduro desarrolle ROP grave desaparezca y que puedan ser excluidos de la red de detección ${ }^{(10,11)}$.

Existen programas emblemáticos de ROP en Latinoamérica; como el de Argentina; que en 
recientes publicaciones muestran como en poco más de una década de funcionamiento el programa nacional logró mejoras en el acceso a la prevención y tratamiento de la ROP, de la misma forma lo logra Chile; que por su geografía a fin de llegar a más lugares incorporó la telemedicina al Programa Nacional de ROP con excelentes resultados ${ }^{(1,4)}$.

De hecho el uso de telemedicina para los programas de ROP se ha extendido en los últimos años; buscando garantizar la cobertura de toda la población de riesgo y buscando subsanar otra problemática; cual es, el número insuficiente de oftalmólogos; esta alternativa ha ayudado a dar otro enfoque a la pesquisa de la ROP; utilizando una cámara retiniana que es transportada por técnicos oftálmicos a unidades neonatales en diferentes lugares donde se capturan imágenes y se transmiten a un oftalmólogo experto que revisa las imágenes y decide sobre la necesidad de tratamiento y establece la frecuencia del seguimiento ${ }^{(12,13,1,1,15)}$. Un modelo multidisciplinario que incluya oftalmólogos, neonatólogos, licenciadas en enfermería y técnicos oftálmicos en función de su disponibilidad es el camino a seguir para aumentar la cobertura de la ROP en países como el nuestro con distintos niveles de acceso a la salud para su población ${ }^{(7)}$.

Desde el Ministerio de Salud Pública de Paraguay, en el año 2016 se establecieron pautas de detección uniformes a fin de no obstaculizar la implementación de un programa de salud pública eficaz para prevenir la ceguera debido a la ROP; estos criterios son: $\mathrm{RN}$ con edad gestacional igual o menor de 34 semanas y/o RN con peso al nacer igual o menor a 1750 gramos; o según criterio médico, los RN mayores a 34 semanas de edad gestacional y/o peso al nacimiento mayor a 1750 gramos que hayan recibido oxígeno suplementario o que tengan otros factores de riesgo asociados; y algo para destacar a fin de evitar oportunidades pérdidas de evaluación; si bien la primera evaluación se debe realizar a las 4 semanas de vida, en algunos casos específicos el primer control oftalmológico se realizará antes, previo al alta del RN, según criterio del médico ${ }^{(16)}$.

La pesquisa de retinopatía del prematuro desde la Fundación Visión se inició en el año 2004; en diciembre del año 2009 posterior al taller de retinopatía de la prematuridad (ROP) desarrollado en Paraguay, organizado por la Fundación Visión en forma conjunta con el CONAVIP (Comité Nacional Visión 2020 Paraguay) y el patrocinio de la OPS (Organización Panamericana de Salud) y el CBM (Christian Blind Mission) se realiza una actualización y análisis de información de ROP como causa de ceguera en Paraguay así como la evaluación de los programas establecidos en el país y se plantean estrategias para mejorar la cobertura a nivel nacional.

El objetivo del presente reporte es describir los resultados de la implementación de un programa de ROP en 7 hospitales de referencia en atención neonatal del país, en relación a la efectividad, accesibilidad y calidad de la atención de la ROP.

\section{MATERIALES Y MÉTODOS}

Estudio Observacional descriptivo, ambispectivo, de casos consecutivos de recién nacidos prematuros con criterios de evaluación internados en las unidades neonatales incluidas en el programa ROP, de una población evaluada en el año 2009 y en los años 2015 al 2019.

\section{Descripción del programa ROP}

Desde la Fundación Visión, el programa de ROP se implementa a mediados del año 2015, por medio de la creación de un equipo multidisciplinario conformado por oftalmólogos; un neonatólogo; técnicos oftálmicos y licenciadas en enfermería.

Si bien el gold standard para la pesquisa de ROP es el examen a través de oftalmoscopia binocular indirecta (OBI); con la finalidad de optimizar las evaluaciones se adquirió una RetCam (Cámara de campo amplio que se utiliza para capturar imágenes de la retina de neonatos prematuros); innovando en telemedicina en esta área en nuestro país.

Se generó un acuerdo con el Ministerio de Salud Pública y Bienestar Social, para poder seguir realizando las evaluaciones en las instituciones públicas que realizan atención a neonatos prematuros. A partir del segundo semestre del 2015 se implementa la recolección de los datos. En los años siguientes gracias a una cooperación internacional 
que permite la ampliación del programa a hospitales del interior del país las pesquisas se amplían primeramente al Hospital Regional de Coronel Oviedo y posteriormente al Hospital Los Angeles de Cuidad del Este. En el 2020 se rediseñaron estrategias; para que a pesar de la situación epidemiológica generada por el Covid 19; las evaluaciones continúen realizándose en tiempo y forma.

Criterios de inclusión: Los RN prematuros con peso de nacimiento menor o igual a 1750 gramos y/o con edad gestacional menor o igual a 34 semanas; ingresados en las unidades neonatales abarcadas por el programa.

Se evaluaron los resultados de implementación a través de los siguientes puntos:

- Adherencia de las unidades neonatales al programa ROP; en sus distintas actividades enfocadas a la prevención, diagnóstico y tratamiento.

- Mejoría de los registros en el tiempo.

Se evaluaron los resultados de accesibilidad a través del siguiente punto:

- Porcentaje de niños evaluados en relación a número de niños con criterios a ser evaluados.

Se evaluaron los resultados de efectividad a través de los siguientes puntos:

- Incidencia de Retinopatía del Prematuro.

- Incidencia de Retinopatía del Prematuro que requirieron tratamiento.

Se evaluaron los resultados de calidad a través de los siguientes puntos:

- Evolución de la frecuencia de Retinopatia del Prematuro en el período de estudio.

- Evolución de la frecuencia de la necesidad de tratamiento.

Los resultados se presentan utilizando estadísticas descriptivas

Aspectos éticos: la información presentada es el resultado de la implementación de un protocolo clínico de evaluación necesario en el manejo y evolución de los recién nacidos prematuros. En el relato del mismo se respetaron los principios éticos aplicados a la investigación: autonomía, beneficencia y justicia.

\section{RESULTADOS}

En la primera evaluación antes de la implementación del programa, se recogieron los datos del período comprendido entre abril 2008 y noviembre 2009 (19 meses). Se evaluaron 202 RN. El 47\% (99/202) presentaron ROP, de los cuales el 18\% (18/99) necesitó tratamiento.

Desde julio del 2015 a diciembre del 2019, 2.397 neonatos prematuros llenaron los criterios para la evaluación de ROP. El 86,8\% (2080/2397) fueron evaluados.

La prevalencia de ROP fue del 20\% (416/2080). El $18,2 \%(76 / 416)$ de los pacientes con $\mathrm{ROP}$ requirieron tratamiento. La procorcion global de ROP grave en la población de estudio fue de 3,6 \% (76/2080).

En el año 2015 el principal resultado estuvo marcado por el aumento en la proporción de las evaluaciones en comparación al primer semestre del 2015 que fue entre el 28 y el $216 \%$; que se observa en la Figura 1.

La distribución por año de los pacientes con criterios de evaluación y el porcentaje de estos que fueron evaluados desde el 2015 al 2019 se muestra en la Figura 2.

El porcentaje de neonatos que presentaron ROP y la necesidad de tratamiento se presenta en la Figura 3.

La frecuencia global en la población de estudio de ROP grave se observa en la Figura 4.

El impacto del Programa en relación al incremento del número de evaluaciones y la frecuencia de ROP se observa en la Figura 5.

De los niños evaluados no se constató ningún niño ciego en el primer control; todos los tratamientos se realizaron entre las 48-72 horas de realizado el diagnóstico. 


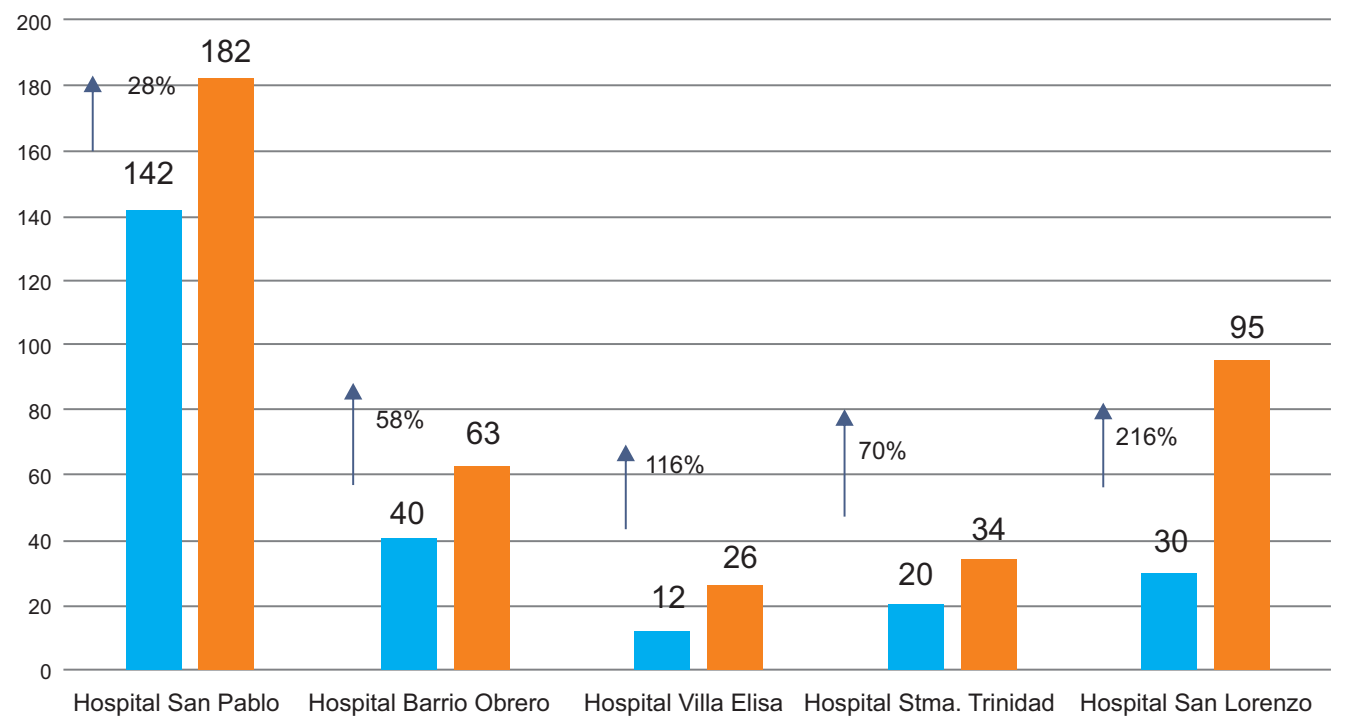

Figura 1. Número de neonatos evaluados en el primer semestre 2015 (barras azules) y número de neonatos evaluados en el segundo semestre 2015 (barra naranja) una vez iniciado el Programa ROP incorporando telemedicina. La proporción del aumento de pacientes evaluados se expresa en porcentajes.

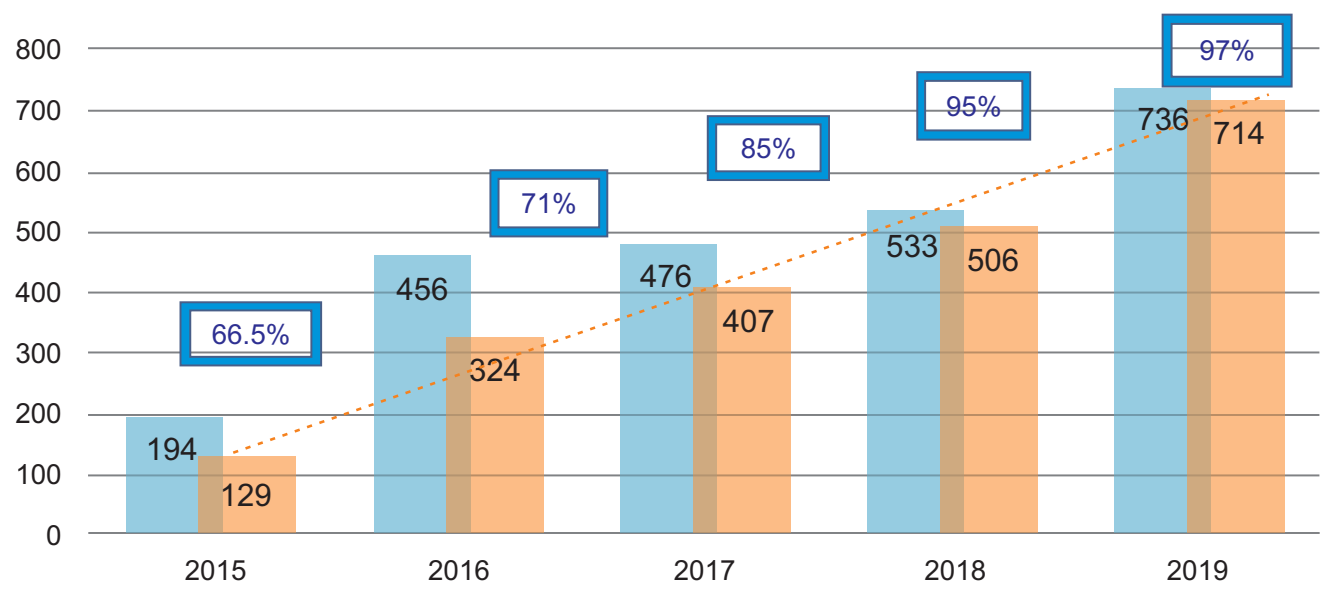

Figura 2. Número de neonatos con criterios de evaluación para ROP (barras naranjas) y numero de evaluados (barra azul) por el programa ROP en los años 2015 a 2019. La recta punteada muestra el incremento de las evaluaciones en porcentaje expresada en porcentajes. 


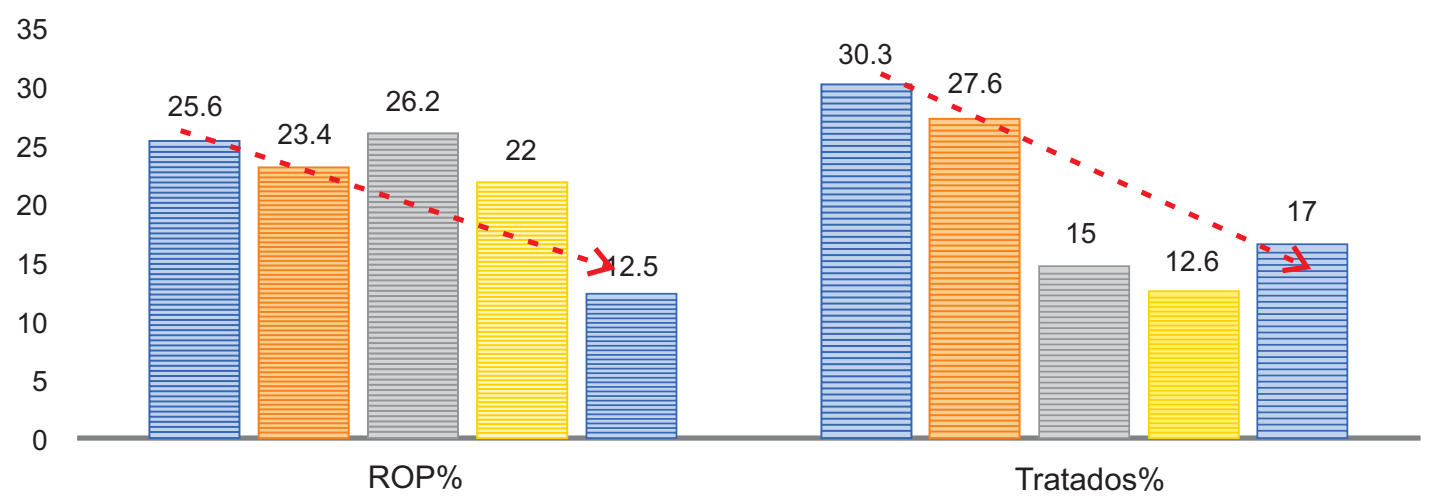

Figura 3. Porcentaje de neonatos del programa con diagnóstico de ROP y porcentaje de ROP que requirieron tratamiento, Período 2015 -2019.

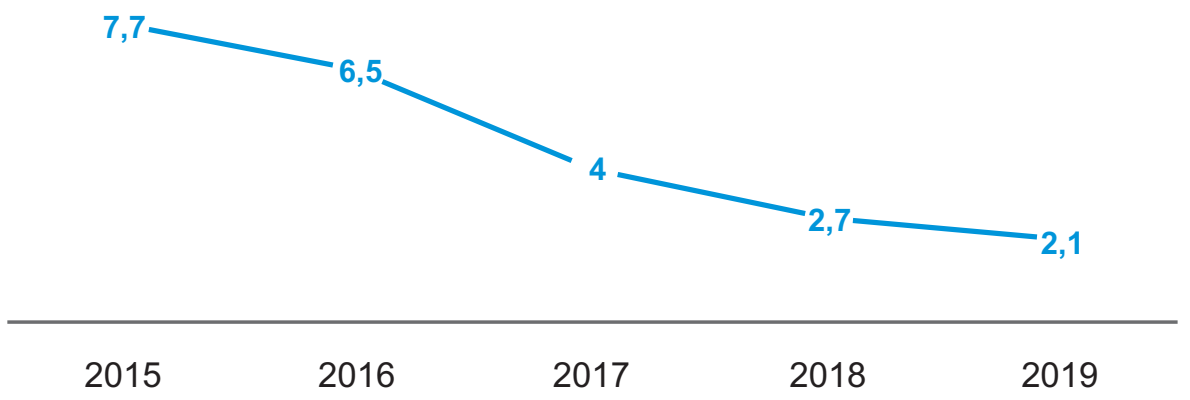

Figura 4. Distribución anual de ROP grave de 2080 neonatos evaluados en el programa ROP - Fundación Visión Período 2015-2019.

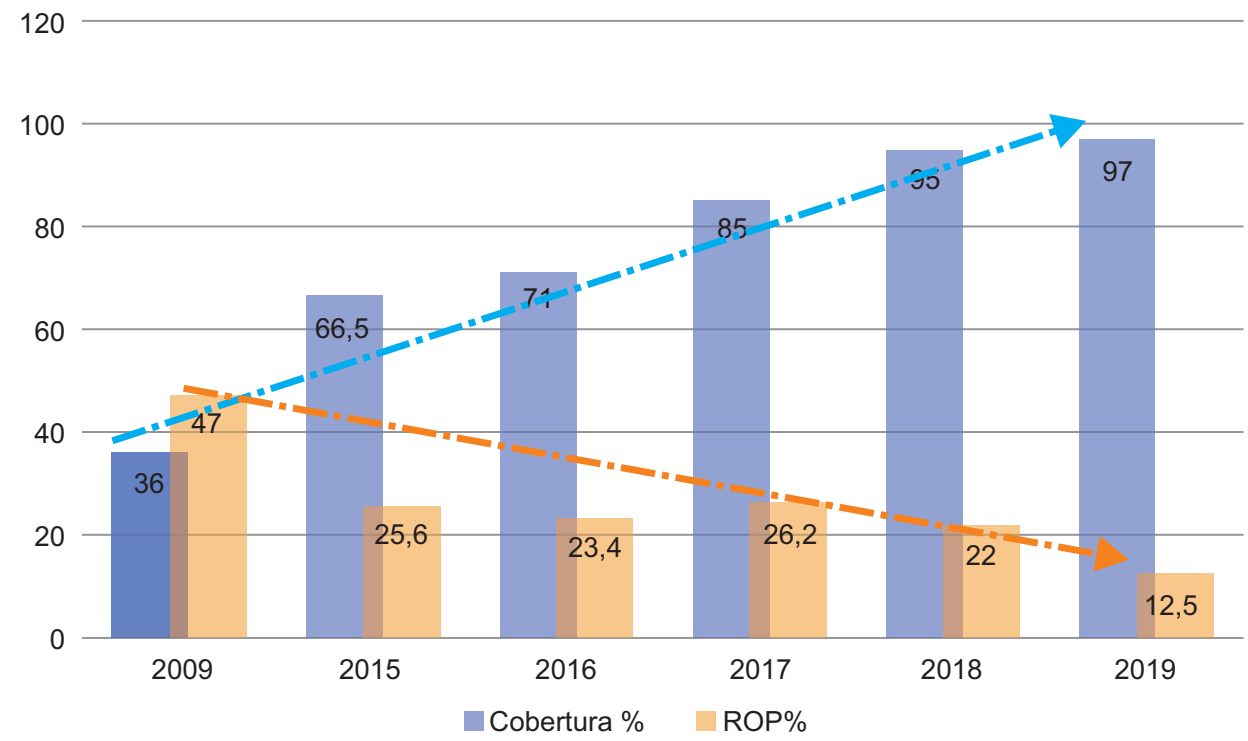

Figura 5. Impacto del Programa ROP, sobre el porcentaje de evaluaciones y la frecuencia de ROP. Período 2015-2016. $N=2080$. 


\section{DISCUSIÓN}

Las mejoras en los indicadores de mortalidad neonatal deben imperiosamente ir acompañadas con mejoras de los indicadores de morbilidad neonatal; $y$ para poder establecer estrategias que mejoren dichos indicadores es clave el registro adecuado a fin de conocer los datos epidemiológicos. Esta es la experiencia de la Fundación Visión con la Retinopatía del Prematuro; que en menor medida pero con igual compromiso ha replicado modelos como el Grupo ROP de Argentina; y recientementela gran experiencia en la India ${ }^{(8,17,18,19)}$. Poder establecer la incidencia de la ROP en una población significativamente representativa de la población de riesgo en nuestro país; establece un punto de corte para poder establecer intervenciones oportunas.

Es fundamental que para establecer programas como estos se entienda la importancia del manejo multidisciplinario; que el equipo de salud tanto médicos como enfermería que están en el continuo de cada niño; participen activamente tanto en la prevención primaria así como en la solicitud de evaluaciones; ningún neonatólogo debe permitir el alta de un paciente con factores de riesgo para ROP; sin una primera evaluación oftalmológica; a fin de evitar oportunidades perdidas de evaluación y riesgos potenciales de ceguera; fue de esta manera que con el programa liderado por la FV se pudo aumentar la cobertura de la población de riesgo llegando a un $98 \%$ y optimizar el seguimiento ${ }^{(20,21)}$.

Se estima que anualmente ocurren 15.000.000 de nacimientos de niños prematuros; de los cuales aproximadamente 2.500 .000 son menores de 32 semanas de edad gestacional; es decir con potenciales factores de riesgo de padecer morbilidades como la
ROP; en nuestro país el $8 \%$ de los nacimientos ocurren de forma prematura, y esto representa aproximada-mente 9000 niños; y la tercera parte (3000 niños) de los mismos tendrán algún factor de riesgo para algún déficit neurosensorial ${ }^{(21,22)}$.

Un manejo adecuado de los mismos evitaría la pérdida de capital humano que implica un soporte familiar tanto para la muerte como para la discapacidad del niño y aumento de la carga de salud y cuidados en edad adulta; en nuestro país la expectativa de vida al nacer es de 74 años; lo que implicaría que si un niño queda ciego antes del año se enfrenta a una vida con 74 años sin visión ${ }^{(21)}$.

A través del programa de ROP de FV se ha evitado la ceguera; de lo que se posee registrado de aproximadamente 100 niños; por tanto con el examen intencionado, bien realizado e innovando a través de telemedicina, fue posible evitar un daño que a la larga tendría un muy alto costo económico, social y familiar. Ante esto la importancia de haber establecido un Programa de ROP desde FV; pudiendo establecer un diagnóstico de la problemática y realizando intervenciones cuyos efectos beneficiosos se vieron con el correr de los años ${ }^{(23,24,25)}$.

\section{CONCLUSIONES}

1- La prevalencia de ROP en el grupo de estudio fue del $20 \%$.

2- La proporción global de ROP grave en la población de estudio fue de $3,6 \%$.

3- Con la introducción de la telemedicina se pudo garantizar mayor cobertura del Programa ROP y optimizar la frecuencia de las evaluaciones subsiguientes. 


\section{REFERENCIAS}

1. Bancalari A, Schade R. Retinopatía del prematuro: actualización en detección y tratamiento. Rev. chil. pediatr. 2020; 91(1):122-130. doi: http://dx.doi.org/10.32 641/rchped.v91i1.1079

2. Ministerio de Salud Pública y Bienestar Social; Dirección de Salud Integral de la Niñez y Adolescencia. Perfil de salud Infantil y Neonatal Paraguay 2011 [Internet]. Asunción: OPS; 2011[Citado 06 Dic. 2020]. Disponible en: http://www.ins.gov.py/wp-content/uploads/2019/09/5.Perfil-de-la-Ni\%C3\%B1ez-Paraguay_compressed.pdf

3. UNICEF Paraguay. Situación del derecho a la salud Materna, infantil y adolescente en Paraguay [Internet]. UNICEF; 2013[Citado 06 Dic. 2020]. Disponible en: https://www.unicef.org/paraguay/informes/situaci\%C3 \%B3n-del-derecho-la-salud-materna-infantil-yadolescente-en-paraguay.

4. Ministerio de Salud Pública y Bienestar Social. Plan Estratégico Institucional del Ministerio de Salud Pública y Bienestar Social correspondiente al periodo 2019-2023 [Documento]. MSPyBS; 2019[Citado 06 Dic. 2020]. Disponible en: https://www.mspbs.gov.py/depen dencias/portal/adjunto/a29109-SO.RSG.N66719 PorelcualseapruebaelPEIdelMSPyBS.pdf

5. Alda E, Lomuto CC, Benítez AM, Bouzas L, Brussa M, Cattaino A, et al. Results of the National Program for the Prevention of Blindness in Childhood by Retinopathy of Prematurity in Argentina (2004-2016). Arch Argent Pediatr. 2018; 116(6):386-393. doi: http://dx.doi.org/ 10.5546/aap.2018.eng.386

6. Clare G, Aeesha M, Nazmun N, Sanjoy K, Linda V, Sarah S, Dupe A. Epidemiology of ROP update - Africa is the new frontier. Semin Perinatol. 2019; 43(6): 317-322. doi: https://doi.org/10.1053/j.semperi.2019.05.002

7. Fierson WM; American Academy of Pediatrics Section on Ophthalmology; American Academy of Ophthalmology; American Association for Pediatric Ophthalmology and Strabismus; American Association of Certified Orthoptists. Screening Examination of Premature Infants for Retinopathy of Prematurity. Pediatrics. 2018; 142(6):e20183061. doi: https://doi.org/10.1542/peds.2018-3061

8. Chawla D, Deorari A. Retinopathy of prematurity prevention, screening and treatment programmes: progress in India. Semin Perinatol. 2019; 43(6):344-347. doi: https://doi.org/10.1053/j.semperi.2019.05.006.
9. Wilson CM, Ells AL, Fielder AR. The challenge of screening for retinopathy of prematurity. Clin Perinatol. 2013 Jun;40(2):241-59. doi: https://doi.org/10.1016/j. clp.2013.02.003

10. Organización Panamericana de la Salud. Guía de práctica clínica para el manejo de la retinopatía de la prematuridad. Washington, D.C.: OPS; 2018.

11. Sivanandan S, Chandra P, Deorari AK, Agarwal R. Retinopathy of prematurity: AIIMS, New Delhi experience. Indian Pediatr. 2016;53(Suppl 2):S123-S128.

12. Barrero-Castillero A, Corwin BK, VanderVeen DK, Wang JC. Workforce Shortage for Retinopathy of Prematurity Care and Emerging Role of Telehealth and Artificial Intelligence. Pediatr Clin North Am. 2020;67(4):725-733. doi: https://doi.org/10.1016/j.pcl.2020.04.012

13. Acevedo-Castellón R, Ramírez-Neria P, García-Franco $\mathrm{R}$. Incidence of retinopathy of prematurity type 1 and type 2 in a regional Hospital of Social Security in the state of Queretaro, Mexico (2017-2018). BMC Ophthalmol. 2019; 19(1):91. doi: https://doi.org/10.1186/s12886-019-1095-0

14. Simkin SK, Misra SL, Han JV, McGhee CNJ, Dai S. Auckland regional telemedicine retinopathy of prematurity screening network: A 10-year review. Clin Exp Ophthalmol. 2019;47(9):1122-1130. doi: https://doi.org/10.1111/ceo.13593

15. Fijalkowski N, Zheng LL, Henderson MT, Wallenstein MB, Leng T, Moshfeghi DM. Stanford University Network for Diagnosis of Retinopathy of Prematurity (SUNDROP): four-years of screening with telemedicine. Curr Eye Res. 2013; 38(2):283-91. doi: https://doi.org/10.3109/ 02713683.2012 .754902

16. Ministerio de Salud Pública y Bienestar Social. Manual de Atención Neonatal [Internet]. 2 ed. MSPyBS; 2016 [Citado 06 Dic. 2020]. Disponible en: https://www.mspbs.gov.py/ dependencias/portal/adjunto/9fac93-MANUALDEATEN CINNEONATAL.AUTORIZADOMSPBSRESOLUCINMI NISTERIALSGN816.pdf

17. Chawla D, Darlow BA. Desarrollo de medidas de calidad en la atención perinatal: prioridad para los países en desarrollo. Indian Pediatr. 2018; 55:797-802. doi: https://doi.org/10.1007/s13312-018-1384-3 
18. Sociedad Panamericana de Retinopatía del Prematuro. Retinopatía del Prematuro [Internet]. Barcelona; 2019[Citado 06 Dic. 2020]. Disponible en: http://www.rop mexico.org.mx/archivos/documentos/libro_rop.pdf

19. Grupo ROP Argentina; Ministerio de Salud. Guía de Práctica Clínica para la prevención, diagnóstico y tratamiento de la retinopatía del prematuro (ROP) [Internet]. Buenos Aires: Ministerio de Salud; 2015 [Citado 06 Dic. 2020]. Disponible en: https://www.sap.org.ar/ docs/comites/cefen/rop_2016.pdf

20. Darlow BA, Gilbert CE, Quiroga AM. Setting up and improving retinopathy of prematurity programs: interaction of neonatology, nursing, and ophthalmology. Clin Perinatol. 2013; 40(2):215-27. doi: https://doi.org/ 10.1016/j.clp.2013.02.006

21. Ministerio de Salud Pública y Bienestar Social; Dirección General de Información Estratégica en Salud. Sub Sistema de Estadísticas Vitales del Ministerio de Salud Pública y Bienestar Social [Internet]. Disponible en: http://ssiev.mspbs.gov.py/
22. Blencowe H, Cousens S, Chou D, Oestergaard M, Say L, Moller AB, et al. Born too soon: the global epidemiology of 15 million preterm births. Reprod Health. 2013;10 Suppl 1(Suppl 1):S2. doi: https://doi.org/10.1186/1742-4755-10S1-S2

23. Blencowe $H$, Cousens S, Oestergaard M, Chou D, Moller A, Rajesh Narwal, et al. National, regional, and worldwide estimates of preterm birth rates in the year 2010 with time trends since 1990 for selected countries: a systematic analysis and implications. The Lancet. 2012; 379(9832): 2162-2172. doi: https://doi.org/10.1016/S01406736(12)60820-4.

24. Quinn GE, Vinekar A. The role of retinal photography and telemedicine in ROP screening. Semin Perinatol. 2019;43(6):367-374. doi: https://doi.org/10.1053/j.sem peri.2019.05.010

25. Wang SK, Callaway NF, Wallenstein MB, Henderson MT, Leng T, Moshfeghi DM. SUNDROP: six years of screening for retinopathy of prematurity with telemedicine. Can J Ophthalmol. 2015 ;50(2):101-6. doi: https://doi.org/10.1016/j.jcjo.2014.11.005 